\title{
Optimization of the Design of Multifrequency Annular Arrays for Very Wide Band Operation
}

\author{
Jorge Topete Garcia ${ }^{1}$, Tomas Gomez Alvarez-Arenas ${ }^{1}$ \\ ${ }^{1}$ Institute for Physical and Information Technologies (ITEFI), \\ Spanish National Research Council (CSIC), \\ Madrid, Spain \\ j.topete@csic.es
}

\begin{abstract}
This paper presents an optimization design strategy for wideband piezoelectric transducers based on a kerfless multifrequency annular array with two elements, made out of a 1-3 connectivity piezo-composite disk. The disk is machined by reducing the thickness within a circular section at the centre of the disk faces, so that two elements are obtained: an inner and thinner disk and an outer ring. They are designed to meet two main criteria: i) both elements present a different resonant frequency, being the inner disk the higher frequency element and ii) the individual frequency bands of the elements overlap to build up an overall frequency response that approximate to the sum of the individual frequency bands. The objective of this research is to determine the optimum configuration of both elements, in particular the relative elevation of the inner disk, so that cross-talk between elements and the appearance of undesired modes is minimized while frequency bandwidth is optimized. Towards this end, the response of different configurations is calculated using a finite element method.
\end{abstract}

Index Terms-Composite materials; piezoelectric transducers; finite element analysis; wideband array.

\section{INTRODUCTION}

Ultrasonic transducers based on piezoelectric materials are normally based on the excitation of geometrical resonances of the piezoelectric element. Although use of resonant devices improves sensitivity of the transducer it also has a negative impact on the frequency bandwidth, which is reduced, especially when compared with other nonresonant solutions (e.g. electrostatic or capacitive transducers [1], [2]). Nonetheless, wide frequency band is a very important transducer feature in many different applications. Examples are found in ultrasonic communications [2], [3], spectral materials characterization [4] and [5], medical image and Non Destructive Testing applications, where bandwidth is also related to spatial resolution. In many cases, frequency bandwidth of piezoelectric transducers can be enhanced by different means: i) selecting piezoelectric materials having higher electromechanical coupling factors [6] or higher mechanical losses or acoustic impedance value closer to that of the medium the transducer is going to be coupled to, ii) using a high impedance backing block, iii) using specially designed matching layers [7], [8] and iv) by acting on the electrical

Manuscript received 16 March, 2016; accepted 3 August, 2016. matching [9]. However, some of these solutions cannot be used for the case of air-coupled transducers, where achieving wideband response is an even more challenging problem due to the huge acoustic impedance difference between the air and the piezoelectric element. Successful solutions in this case have been the use of multiple matching layers [10][13], wider bandwidth piezoelectric materials (like 1-3 connectivity composites based on PMN-PT single crystals) [14] and active materials with acoustic impedance closer to the air, examples are the use of ferroelectret films [13], [15], [16] and PVDF [17].

In spite of these efforts, $6 \mathrm{~dB}$ bandwidth of air-coupled piezoelectric transducers is always below $100 \%$, and further improvements in this direction can only be achieved by adopting novel and alternative approaches. One example is the use of multifrequency arrays where different elements are designed to operate at different frequencies, but their frequency bands overlap giving rise to a much larger overall frequency bandwidth [18]. However, a design able to reduce cross-talk between elements and to homogenize the acoustic fields of the different elements is necessary.

\section{Model AND Methods}

The starting point of this work is the design proposed in [18] that consists of a kerfless annular multifrequency array disk with two elements. It is made out of a 1-3 connectivity piezocomposite disk (random distribution of PZT fibers in an epoxy matrix by Smart Materials Inc.). A 1-3 connectivity composite disk has been chosen because this type of material permits to reduce the cross-talk between thickness and radial modes which is important to reduce the cross-talk between the two elements in the annular array configuration proposed here. Fig. 1 shows a schematic view of the piezocomposite annular array with the two elements: centre disk and external ring. In addition, internal geometry of the 1-3 connectivity composite is also depicted.

As explained in [18] (see also Fig. 1), the piezocomposite disk is machined to obtain a kerfless array with two elements: an external ring (tuned to $250 \mathrm{kHz}$ ) and a thinner internal disk (tuned to $500 \mathrm{kHz}$ ). Diameter of the outer ring in $25 \mathrm{~mm}$ and diameter of the inner disk is $12 \mathrm{~mm}$. Unlike in [18], where both elements presented the same elevation $(\mathrm{h}=$ 0 ), now this elevation is changed with the purpose to find out if there is an optimum value for the parameter $h$. The main 
idea is then to determine if matching the nodal planes of both elements in the annular array may enhance the synergy of both elements and have some positive influence on achieving a more uniform displacement of both elements and a more reduced cross-talk. In particular, to determine if there is an optimum arrangement of the elements, able to maximize the surface displacement in the case of emission and the charge density in the case of reception.

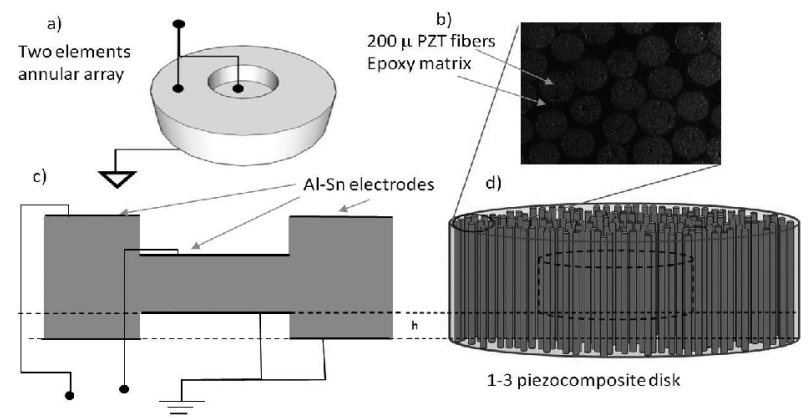

Fig. 1. 3D View of the piezocomposite array and its two elements: inner disk and outer ring used for the study. Polarization is in the thickness direction (a); Surface image of the composite revealing the PZT fibers and the epoxy matrix (b), cross-section view of the array showing electrodes, electrical connections and relative elevation (c) of both elements (h); representation of the original 1-3 connectivity piezocomposite disk (d).

Several configurations have been considered; the array response in each case is calculated using a Finite Element Method (FEM). Use of a FEM approach to these kind of problems is normally adopted in this field. In this case, it is required by the need to have a 3 dimensional model, where the main reason is the need to consider the cross-talk between elements when operating in thickness mode. As this cross-talk takes place through the generation of radial modes produced by coupling of thickness to lateral vibrations through the material Poisson's ratio a full 3D approach is necessary. The Finite Element Method model is built using the commercial package COMSOL Multiphysics (by COMSOL Inc.) and materials parameters are obtained from the manufacturer of the piezocomposite (Smart Materials) and from data obtained from size and density measurements and from measurements of the frequency location of different resonant modes in the electrical impedance vs. frequency response.
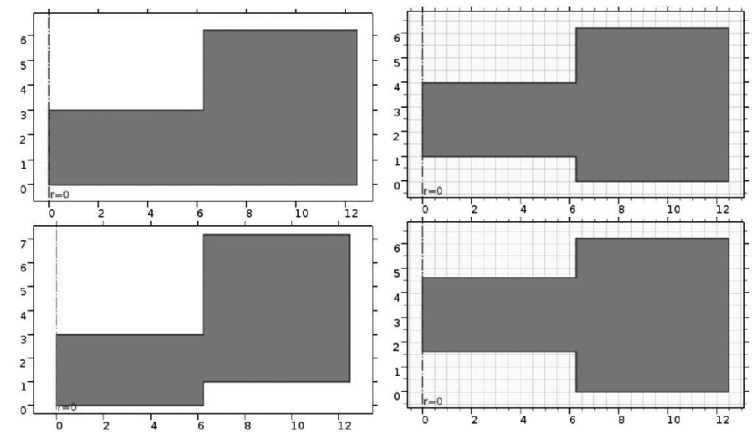

Fig. 2. Cross-section of one half of the machined disk showing the two elements (inner disk: high frequency element and the external ring: low frequency element) and the different geometric arrangements (elevations) of the array elements subject to simulation.

Figure 2 shows a cross-section view (only the half required to generate the solid of revolution that corresponds to the actual array geometry, see Fig. 1) of the different array geometries proposed in this study (with different elevations for each element). The relative positions of the array elements are changed with the purpose to change the relative position of the nodal planes of the thickness resonances of the two different elements, with the idea to test if the case where both nodal planes coincide actually corresponds to the optimum design.

\section{RESULTS}

Figure 3 shows the FEM calculated electrical impedance of the array (both elements parallel connected) for the different configurations shown in Fig. 2. The two different thickness modes can be clearly observed at $250 \mathrm{kHz}$ and $500 \mathrm{kHz}$, which correspond to the external ring and the inner disk, respectively. In addition, resonance $\left(f_{r}\right)$ and antiresonance $\left(f_{a}\right)$ frequencies for these thickness modes of each element are also indicated in Fig. 3. In spite of the use of a 1-3 connectivity composite, radial modes are still to be observed at lower frequency (below $150 \mathrm{kHz}$ ), although they are quite attenuated and their influence on thickness modes is expected to be small.

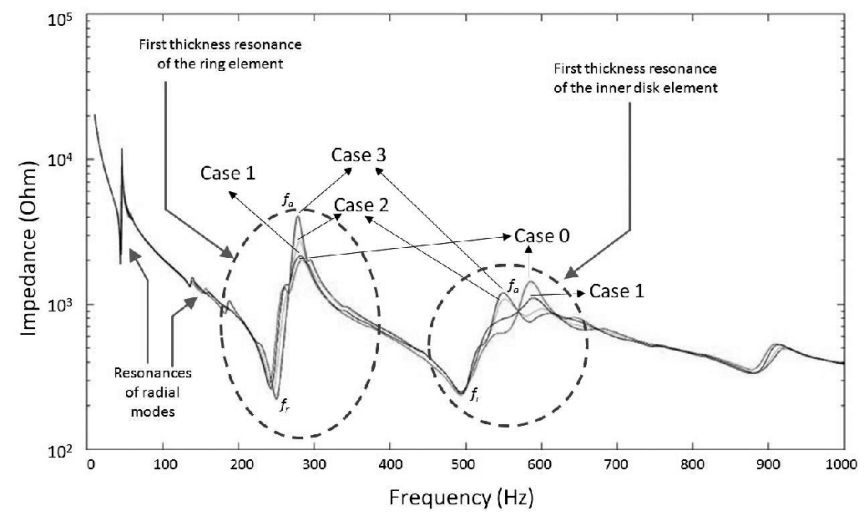

Fig. 3. FEM calculated electric impedance for the four different geometric arrangements shown in Fig. 2.

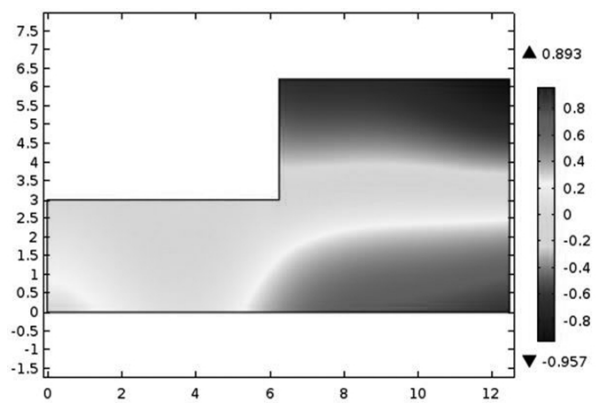

a) a)

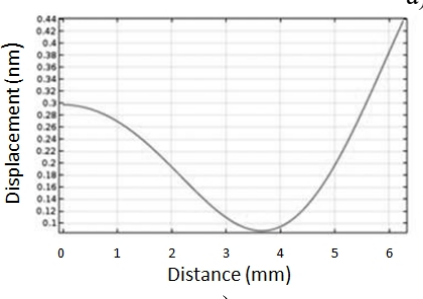

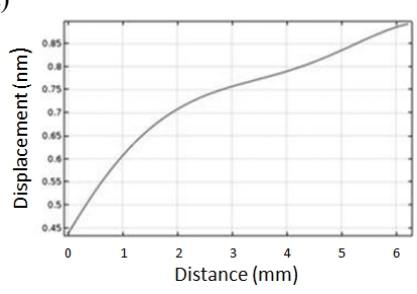

b)
Fig. 4. Case 0. Excitation at $250 \mathrm{kHz}$ ( $f_{r}$, outer ring element): (a) distribution of displacements along the direction normal to the thickness over the array cross-section; (b) displacement along the thickness direction at the surface $(\mathrm{x}=0)$ of the inner disk; $(\mathrm{c})$ displacement along the thickness direction at the surface $(\mathrm{x}=0)$ of the outer ring. 
The point of the simulations is to find the most advantageous value of $h$, towards this end analyse the response of the array both in emission and reception operation modes. To study the response in emission ode we analyse the surface displacement at $f_{r}$, while the evaluation in reception mode is performed by the analysis of the and charge density at $f_{a}$ generated by a given surface displacement.

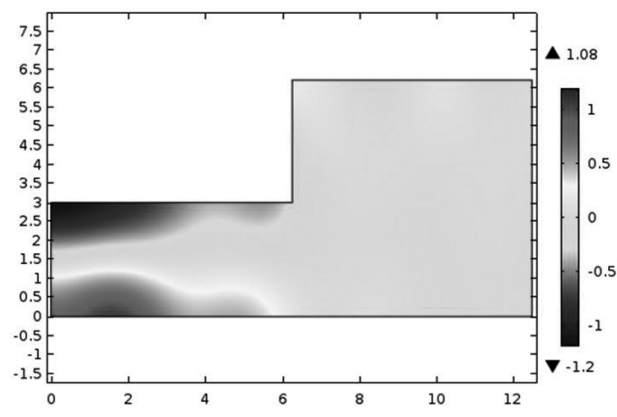

a)

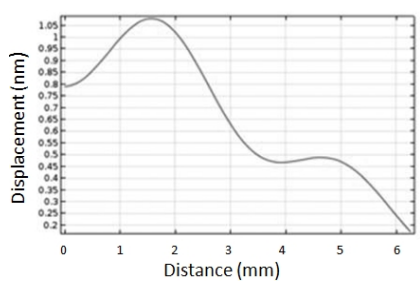

b)

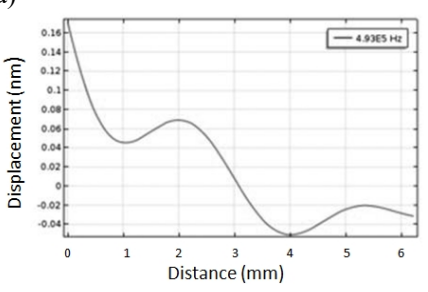

c)
Fig. 5. Case 0. Excitation at $500 \mathrm{kHz}\left(f_{r}\right.$, inner disk element): (a) distribution of displacements along the direction normal to the thickness over the array cross-section; (b) displacement along the thickness direction at the surface $(\mathrm{x}=0)$ of the inner disk; (c) displacement along the thickness direction at the surface $(\mathrm{x}=0)$ of the outer ring.

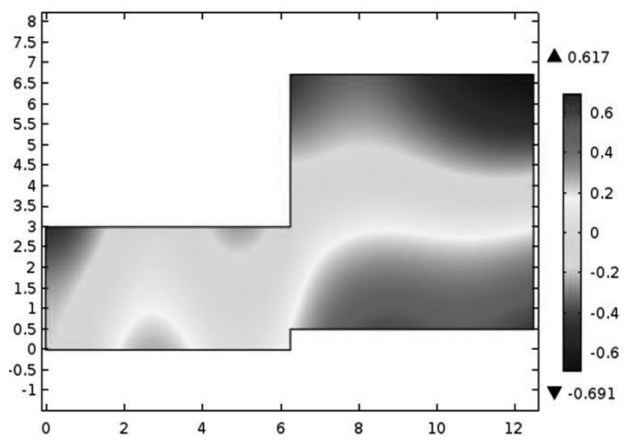

a)

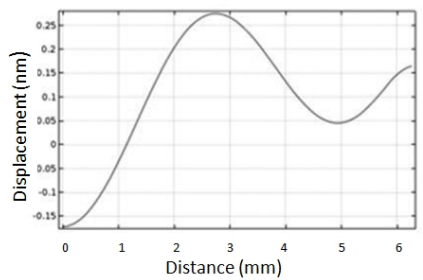

b)

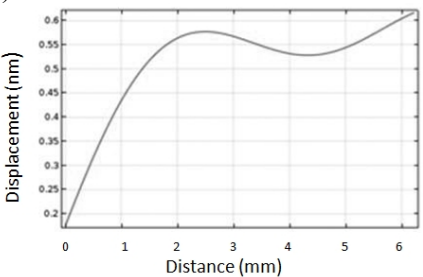

c)
Fig. 6. Case 1. Excitation at $250 \mathrm{kHz}\left(f_{r}\right.$, outer ring element): (a) distribution of displacements along the direction normal to the thickness over the array cross-section; (b) displacement along the thickness direction at the surface $(\mathrm{x}=0)$ of the inner disk; (c) displacement along the thickness direction at the surface $(\mathrm{x}=0.5)$ of the outer ring.

First the behaviour of the array as transmitter is analysed. In this case, electrical voltage is applied across composite terminals and the mechanical response in terms of generated particle displacement at $f_{r}$ is calculated. Figure 4-Fig. 11 show the distribution of the amplitude of the displacement in the thickness direction over the array cross-section and the normal displacement at the radiating surface.

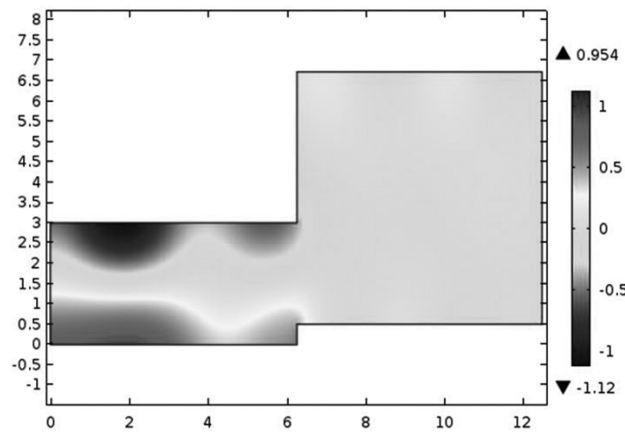

a)

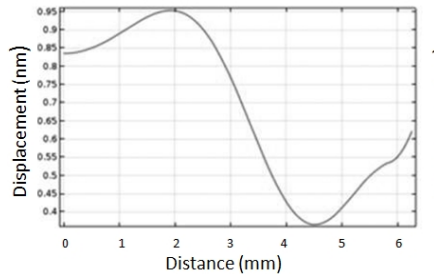

b)

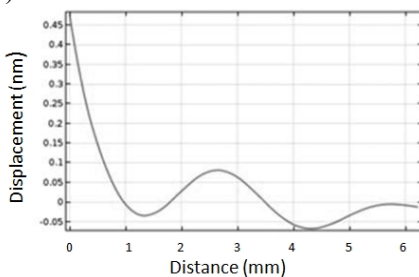

c)
Fig. 7. Case 1. Excitation at $500 \mathrm{kHz}\left(f_{r}\right.$, inner disk element): (a) distribution of displacements along the direction normal to the thickness over the array cross-section; (b) displacement along the thickness direction at the surface $(\mathrm{x}=0)$ of the inner disk; (c) displacement along the thickness direction at the surface $(\mathrm{x}=0.5)$ of the outer ring.

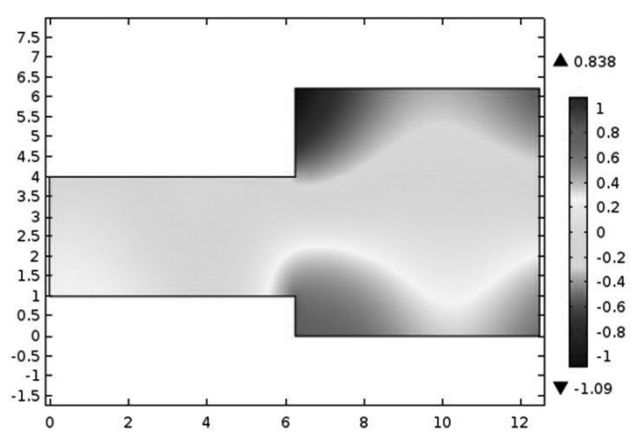

a)

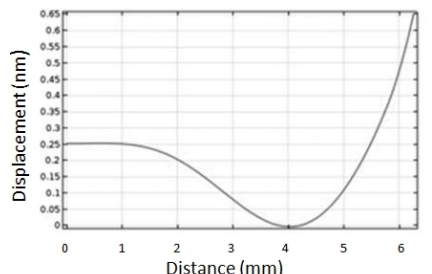

b)

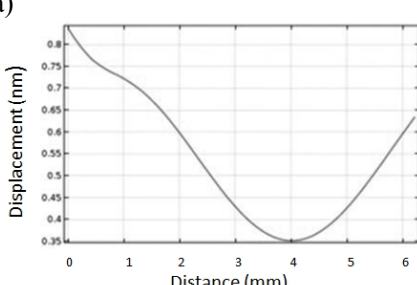

c)
Fig. 8. Case 2. Excitation at $250 \mathrm{kHz}$ ( $f$, outer ring element). (a) distribution of displacements along the direction normal to the thickness over the array cross-section; (b) displacement along the thickness direction at the surface $(\mathrm{x}=1.0)$ of the inner disk; (c) displacement along the thickness direction at the surface $(\mathrm{x}=0)$ of the outer ring.

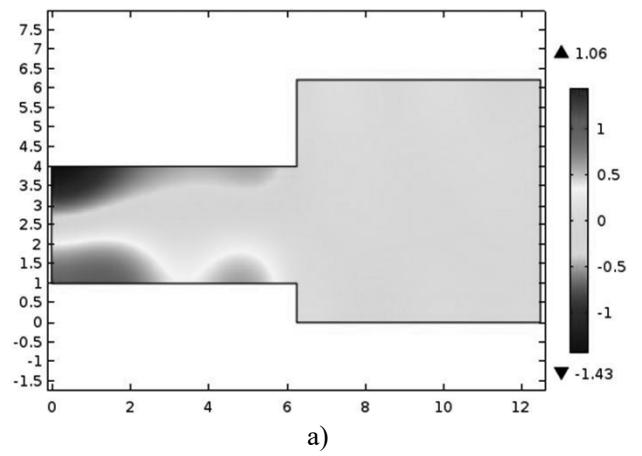




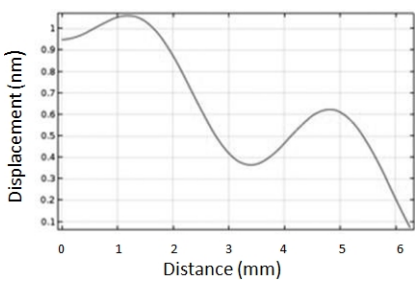

b)

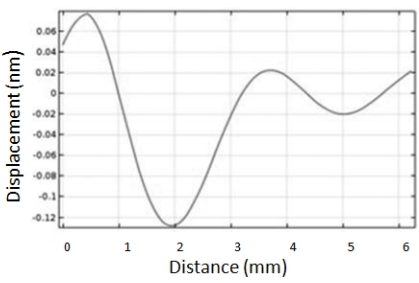

c)
Fig. 9. Case 2. Excitation at $500 \mathrm{kHz}\left(f_{r}\right.$, inner disk element): (a) distribution of displacements along the direction normal to the thickness over the array cross-section; (b) displacement along the thickness direction at the surface $(\mathrm{x}=1.0)$ of the inner disk. (c) displacement along the thickness direction at the surface $(\mathrm{x}=0)$ of the outer ring.

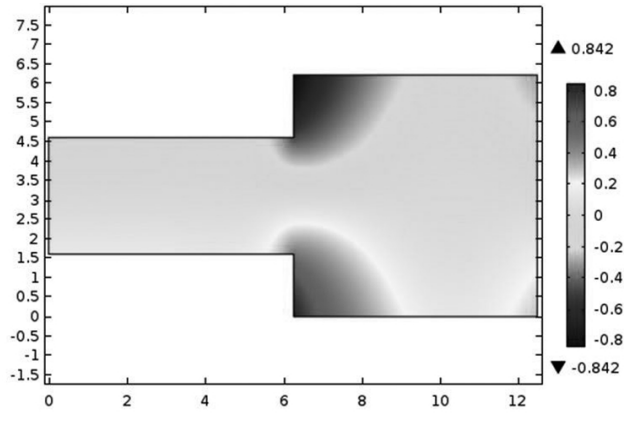

a)

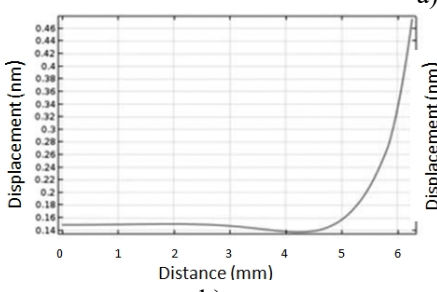

b)

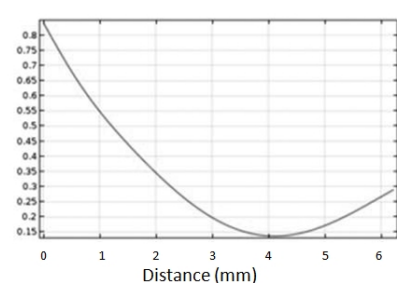

c)
Fig. 10. Case 3. Excitation at $250 \mathrm{kHz}\left(f_{r}\right.$, outer ring element): (a) distribution of displacements along the direction normal to the thickness over the array cross-section; (b) displacement along the thickness direction at the surface $(\mathrm{x}=1.5)$ of the inner disk; (c) displacement along the thickness direction at the surface $(\mathrm{x}=0)$ of the outer ring.

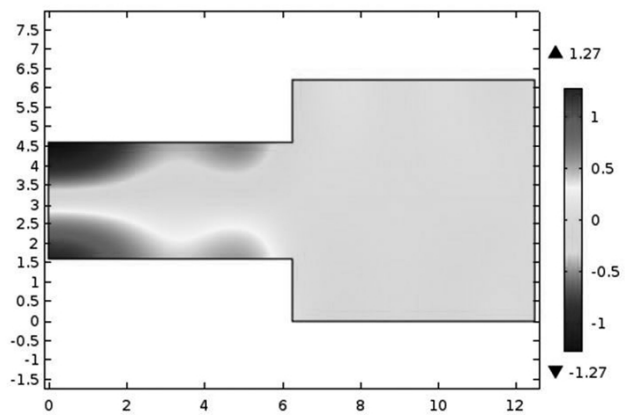

a)

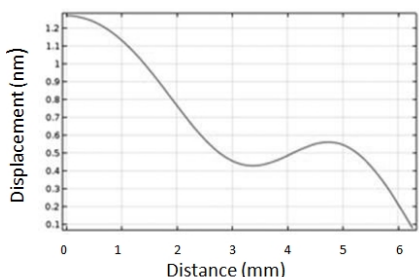

b)

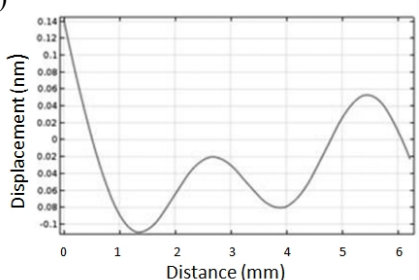

c)
Fig. 11. Case 3. Excitation at $500 \mathrm{kHz}\left(f_{r}\right.$, inner disk element): (a) distribution of displacements along the direction normal to the thickness over the array cross-section; (b) displacement along the thickness direction at the surface $(\mathrm{x}=1.5)$ of the inner disk; $(\mathrm{c})$ displacement along the thickness direction at the surface $(\mathrm{x}=0)$ of the outer ring.

To be able to propose a best array configuration candidate for the emission mode a figure of merit based on the mean value (averaged over the radiating surface of each element) of the surface displacement (normal to the radiating surface), along with the standard deviation, is proposed. The mean provides a measure of the peak energy radiated by the element and the standard deviation provides a measure of how close is the observed behaviour to a piston-like ideal behaviour (as standard deviation is expected to be zero for the ideal piston-like displacement pattern). These results are shown in Fig. 12 and Fig. 13.

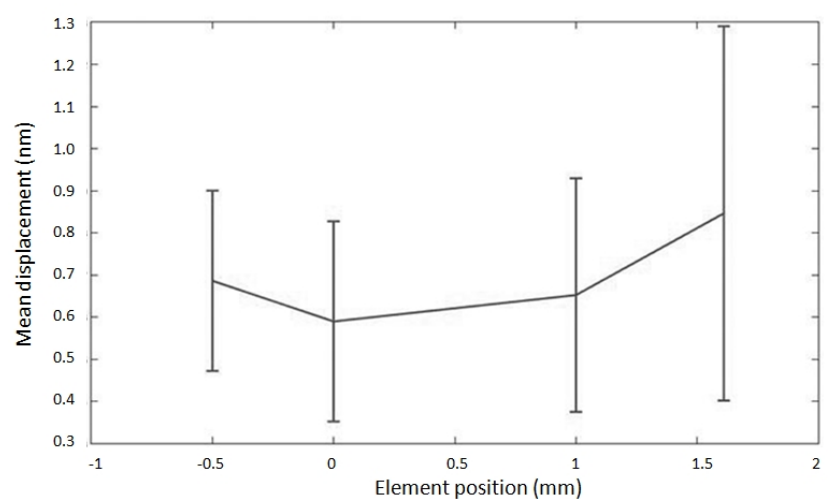

Fig. 12. Averaged displacement and standard deviation over the surface of the outer ring element radiant surface vs relative elevation of the inner disk.

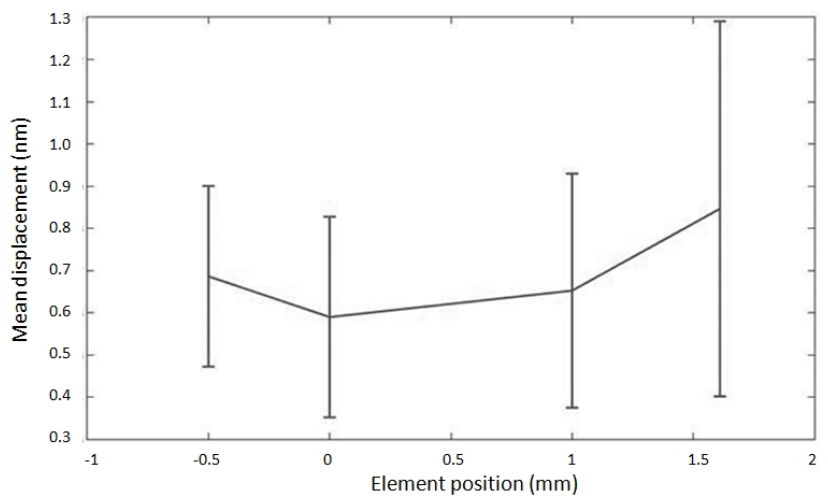

Fig. 13. Averaged displacement over the surface of the inner disk of the Internal element radiant surface vs relative elevation.

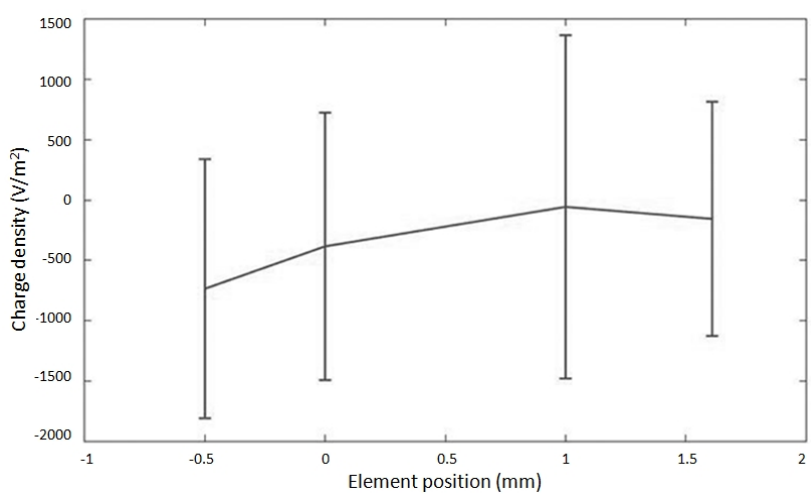

Fig. 14. Averaged charge density and standard deviation over the surface of the outer disk element radiant surface vs relative elevation of the inner disk.

In a similar way, for reception operation mode, the proposed figure of merit is based on the averaged (over the surface of each element) electrical charge generated at the surface of each element (upon normal incidence of a normalized plane wave) and the standard deviation. As before, mean value and standard deviation are representative of the peak and the piston-like response, respectively. Results are shown in Fig. 14 and Fig. 15. 


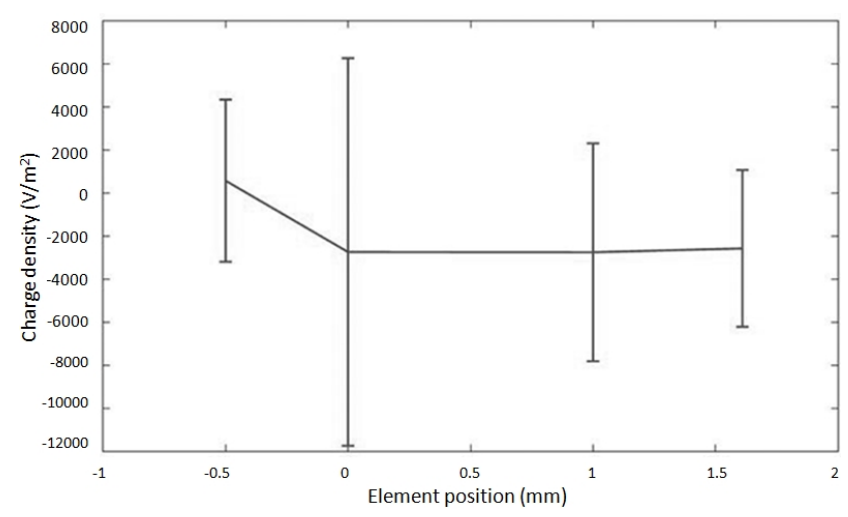

Fig. 15. Averaged charge density and standard deviation over the surface of the inner disk element radiant surface vs relative elevation of the inner disk.

\section{CONCLUSIONS}

We can draw some conclusions regarding the best suited emitter from the data gathered. The different geometric arrangements show a variation in the mean displacement of the surface but also a dispersion of the data, meaning that the movement along the surface is not regular due to some lateral mode interference. For example, Fig. 4 and Fig. 5 (Case 0) present a rather piston-like vibration of the surface and very little cross-talk. In the exterior element we can see an improvement, even regarding the uniformity of the displacement, in the case where the centre of the elements is aligned (case 3), however, in this case the behaviour significantly deviates away from the piston-like desired pattern, which is revealed by the larger standard deviation in Fig. 12. This is not happening for the interior element, although the mean displacement grows the modal interference also grows. It is clear that the geometric approach to fine tuning the modes present in the transducer is promising, more strategies can be implemented aimed to suppress or attenuate the lateral modes.

Looking at the receiver data, we can point out some candidates but the data is too disperse to be a clear winner. Some change in the electrode geometry can lead to a reduction of charge density distribution.

The case 0 shows a promising compromise, the exterior element performs a little worse $(0.1 \mathrm{~nm})$ than the case 3 but the interior element gets an improvement in terms of mean displacement and uniformity. Another added advantage is due to the radiant surface having one of the elements protruding, this protrusion gives room to accommodate the matching layers needed to couple the transducer to air, given that the matching layer of the inner element is thinner than the one needed for the outer element the arrangement can be fine adjusted in order to get a planar radiant surface which improves the emitted wavefront characteristics of the transducer.

\section{REFERENCES}

[1] M. I. Haller, B. T Khuri-Yakub, "A surface micromachined electrostatic ultrasonic air transducer", in IEEE Ultrasonics Symposium, 1994, pp. 1241-1244. [Online]. Available: http://dx.doi.org/10.1109/ultsym.1994.401810

[2] C. Li, D. A Hutchins, R. J. Green, "Short-range ultrasonic communications in air using quadrature modulation", IEEE Trans. Ultrason. Ferroelectr. Freq. Control, vol. 56, no. 10, pp. 2060-2072, 2009. [Online]. Available: http://dx.doi.org/10.1109/TUFFC.2009. 1289

[3] J. Zhang, Z. Huang, X. Liu, "Acoustic communication in wireless sensor networks", in Wireless Sensor Networks, pp. 1-8, 2005.

[4] T. E. G. Alvarez-Arenas, F. Montero, M. Moner-Girona E. Rodriguez, A. Roig, E. Molins, "Viscoelasticity of silica aerogels at ultrasonic frequencies", App. Phys. Lett., vol. 81, no. 7, p. 1198, 2002. [Online]. Available: http://dx.doi.org/10.1063/1.1499225

[5] T. E. G. Alvarez-Arenas, "Air-coupled ultrasonic spectroscopy for the study of membrane filters", J. Membr. Sci., vol. 213, no. 1-2, pp. 195-207, 2003. [Online]. Available: http://dx.doi.org/10.1016/ S0376-7388(02)00527-6

[6] S. Cochran, M. Park, P. Marin-Franch, "Ultrabroadband single crystal composite transducers for underwater ultrasound", in IEEE Ultrasonics Symp., 2005, pp. 231-234. [Online]. Available: http://dx.doi.org/10.1109/ultsym.2005.1602838

[7] C. S. Desilets, J. D. Fraser, G. S. Kino, "The design of efficient broad-band piezoelectric transducers", IEEE Trans. Sonics Ultrason., vol. 25, no. 3, pp. 115-125, 1978. [Online]. Available: http://dx.doi.org/10.1109/T-SU.1978.31001

[8] J. A. Hossack, B. A. Auld, "Improving the characteristics of a transducer using multiple piezoelectric layers", IEEE Trans. Ultrason., ferroelec. Freq. Contr., vol. 40, no. 2, pp. 131-139. 1993. [Online]. Available: http://dx.doi.org/10.1109/58.212561

[9] Z. Zhao, "Broad-band electrical matching of transducers for acoustic microscopy", Ultrasonics, vol. 25, no. 2, pp. 95-99, 1987. [Online]. Available: http://dx.doi.org/10.1016/0041-624X(87)90067-9

[10] T. Gudra, K. J. Opielinski, "Influence of acoustic impedance of multilayer acoustic systems on the transfer function of ultrasonic airborne transducers", Ultrasonics, vol. 40 , no. 1-8, pp. 457-463, 2002.

[11] T. E. G. Alvarez-Arenas, "Acoustic impedance matching of piezoelectric transducers to the air", IEEE Trans. Ultrason., Ferroelec. Freq. Contr., vol. 51, no. 5, pp. 624-633, 2004. [Online]. Available: http://dx.doi.org/10.1109/TUFFC.2004.1320834

[12] S. P. Kelly, G. Hayward, T. E. G Alvarez-Arenas, "Characterization and assessment of an integrated matching layer for air-coupled ultrasonic applications", IEEE Trans. Ultrason. Ferroelect., Freq., Contr. vol. 51, no. 10, pp. 1314-1323, 2004. [Online]. Available: http://dx.doi.org/10.1109/TUFFC.2004.1350960

[13] T. E. G. Alvarez-Arenas, "Air-coupled piezoelectric transducers with active polypropylene foam matching layers", Sensors, vol. 13, no. 5, pp. 5996-6013, 2013. [Online]. Available: http://dx.doi.org/ $10.3390 / \mathrm{s} 130505996$

[14] T. E. G. Alvarez-Arenas, T. R. Shrout, S. J. Zhang, H. J. Lee, "Aircoupled transducers based on 1-3 connectivity single crystal comp", in IEEE Int. Ultrasonics Symp., Dresden, 2012.

[15] J. Doring, J. Bartusch, U. Beck, A. Erhard, "EMFIT Ferroelectret film transducers for non-contact ultrasonic testing", European Conf. NDT, 2006.

[16] J. L. Ealo, J. Camacho, C. Fritsch, F. Seco, J. Roa, “A fabrication procedure for airborne ultrasonic phased arrays based on cellular electromechanical film", in IEEE Ultrasonics Symposium, 2008, pp. 891-894. [Online]. Available: http://dx.doi.org/10.1109/ultsym. 2008.0215

[17] M. Toda, "Phase-matched air ultrasonic transducers using corrugated PVDF film with half wavelength depth", IEEE Tran. Ultrason., Ferroelec. Freq.Control, vol. 48, no. 6, pp. 1568-1574, 2001. [Online]. Available: http://dx.doi.org/10.1109/58.971708

[18] J. Topete, T. E. Gomez Alvarez-Arenas, "Annular multifrequency piezoelectric array for enhanced wideband ultrasonic response", in IEEE Sensors, pp. 102-105, 2014. [Online]. Available: http://dx.doi.org/10.1109/icsens.2014.6984943 